\title{
Significance of Vitamin A (Retinol) in Ageing
}

\author{
T. Basu ${ }^{1, *}$ and A. Doeve ${ }^{2}$
}

${ }^{1}$ Department of Agricultural, Food \& Nutritional Science University of Alberta, Edmonton, Alberta, Canada

${ }^{2}$ Department of Health Education, Kaiser Permanente Riverside, California, USA

\begin{abstract}
Summary: In recent years, studies involving animal models, case reports, and some epidemiological studies have indicated that an accumulation of vitamin $\mathrm{A}$ in the liver is linked to low bone mineral content, fracture risk, and hence osteoporosis. While vitamin A in its physiological level acts as an inducer of bone matrix protein (osteocalcin) and matrix GLA protein in the osteoblast cells (bone formation), excess reinoic acid (a metabolic product of vitamin A) supresses osteoblastic activity and stimulates osteoclast formation (bone degradation). There also appear to have an antagonistic or synergistic interactions between vitamins A and D, influencing bone metabolosm; such effects are dose related. These effects could be of concern, especially for the older persons, because of the age-associated cumulation of vitamin A in the liver. The vitamin A status in elderly population, however, has not been as well documented as in an earlier age. In effect, the Food and Nutrition Board of National Academy's Institute of Medicine has not made any differenciation in it's recommendation for the Dietary Reference Intake (DRI) of vitamin A between 14 years and $>70$ years old individuals. This report will delineate the ageing differences in metabolic availability of vitamin A and that such differences are independent of its intake. The report will also attempt to formulate a hypothesis that in old age, deficiency of vitamin A is not a concern but its potential toxicity involving bone.
\end{abstract}

Keywords: Vitamin A, ageing; toxicity, osteoporosis, vitamin D metabolism, oxidative stress.

\section{INTRODUCTION}

Vitamin A (retinol) plays a major role in vision, cell growth and maintenance of the integrity of epithelial cells. The role of this vitamin in the promotion of growth and differentiation of epithelial tissues makes it an important nutrient during development of growth, reproduction, bone development, and immunity. Vitamin A deficiency and its consequences in early life have thus been well documented. The vitamin A status in elderly population, however, has not been as well documented as in earlier age. In effect, the Food and Nutrition Board of National Academy's Institute of Medicine has not made any differenciation in it's recommendation for the Dietary Reference Intake (DRI) of vitamin A between 14 years and $>70$ years old individuals [1]; this is true for both males and females. This report will delineate the ageing differences in metabolic availability of vitamin A and that such differences are independent of its intake. The report will also attempt to formulate a hypothesis that in old age, deficiency of vitamin A is not a concern but its potential toxicity involving bone.

\section{VITAMIN A METABOLISM}

Pre-formed vitamin A (retinol) is present in food essentially as long-chain fatty acid esters (e.g., retinyl palmitate), which are exclusively of animal origin. The retinyl esters are hydrolyzed to retinol in the intestine before absorption [2]. In the enterocytes, retinol is reesterified and incorporated into

\footnotetext{
*Address correspondence to this author at the Department of Agricultural, Food \& Nutritional Science University of Alberta, Edmonton, Alberta, Canada; Tel: 780487 7133; Fax: 780492 4265; E-mail: Tapan.Basu@ales.ualberta.ca
}

chylomicrons together with triglycerides. The chylomicrons carry retinyl esters to the liver, where it is stored predominantly in the stellate cells. Prior to transport of retinol to the target tissues, the hepatic retinyl esters are hydrolyzed to retinol, which then binds to its carrier, retinol-binding protein (RBP), which is a zinc containing protein, synthesized in the liver [3]. The retinol-RBP complex is then released into the circulation, where it forms a complex with another protein, transthyretin (TTR), as a 1:1:1 molar ratioo. At target tissues, TTR is released and RBP facilitates the uptake of retinol by RBP receptors. Retinol binds with cellular retinolbinding protein (cRBP), which facilitates the transport of retinol. Retinol may be esterified and stored or may be converted to more active metabolites, such as retinoic acid.

\section{AGE- ASSOCIATED CHANGES IN PLASMA AND LIVER LEVELS OF VITAMIN A}

Using male normal rats of different ages, plasma levels of retinol have been reported to be significantly decreased, while its hepatic concentrations are increased in $32-35$ months old compared with those of the 4-19 months old rats [4]. An evaluation of the hepatic store of vitamin A in humans is difficult since the only way to obtain such data is from liver samples collected during autopsy. There has been, however, an early study [5], which reported vitamin A concentrations in various human tissues, including liver, that were collected at autopsy from five geographic areas in the United States (California, Iowa, Missouri, Ohio and Texas). This report provided liver vitamin A concentrations by age groups but without any comments on the age-associated changes. Re-examination of these data has clearly shown 
that the hepatic concentrations of vitamin A are increased with increasing age (Table 1), agreeing the experimental results [4].

Table 1. Vitamin A Concentration in Human Liver by Age Groups*

\begin{tabular}{|c|c|c|}
\hline Age (Year) & No. of Subjects & Vitamin A, ug/g (mean \pm SD) \\
\hline \hline $11-30$ & 35 & $96 \pm 35$ \\
\hline $31-50$ & 70 & $110 \pm 28$ \\
\hline $51-70$ & 136 & $140 \pm 20$ \\
\hline $71-90$ & 79 & $202 \pm 18$ \\
\hline
\end{tabular}

*modified from Rer. [5].

Plasma (or serum) retinol, because of its easy accessibility, is the most commonly used biochemical index for the vitamin status in humans. The plasma values, however, do not reflect body stores of vitamin A because the plasma contains only $1 \%$ of the total body reserve and the values are homeostatatically controlled. Plasma vitamin A is changed only when the hepatic store is severely depleted or excessively high. In addition, factors such as low protein status, acute catabolic status, and decreased hepatic function are all known to affect the RBP production and therefore the metabolic availability of vitamin A from its hepatic stores [6]. Plasma vitamin A levels alone do not reveal if there is an impairement in its metabolic availability. These values can be interpreted better, however, if they are measured along with RBP [7]. This is because the transport of retinol from the liver to the target issues is dependent upon the carrier protein. Thus in vitamin A deficient rats, the serum concentration of RBP is reduced while it's level is increased in the hepatic tissue, and that this scinario is riversed when vitamin $\mathrm{A}$ is administered to this deficient rats [8]. Normally, such effects do not appear to be caused by synthesis of RBP, rather the availability of vitamin $\mathrm{A}$ in concommitant with the need for vitamin A to be transported to the blood as a function of homeostatic regulation of circulatory level of vitamin A. In old age, however, this effect does not appear to be primarily caused by it's synthesis since the RBP concentration in the hepatic tissue is considerably high, compared with that of the younger age. Furthermore, when vitamin A is administered to rats deficient in the vitamin, the RBP concentration in the liver decreases concomitantly with an increase in serum concentration. The underlying mechanism for homeostasis of plasma retinol is essentially mediated through syn- thesis and secretion of RBP. However when there is an excess intake of the vitamin for a long period of time the hepatic storage capacity and the retinol-binding capacity of RBP are exceeded to their limits.

Using experimental old rats the hepatic store of vitamin A has been shown to be negatively associated with it's carrier protein [9]. Thus the hepatic storage of retinol concentration is considerably higher, while RBP mRNA is lower in 18-20 months old than in 2-10 months old rats (Table 2). Wheather this associationship in old age is counterbalenced by an increased turnover rate of RBP or whether this reflects a physiologically diminished demand for vitamin A, cannot be ascertained at this point. Dawson and his associates [9] have, however, shown that unlike the RBP expression the cRBP expression remains unaffected in old age, reflecting a normal transport of vitamin A to its target cells.

The overall evidence clearly suggests that vitamin A, as a nutrient, is not a concern for the older population. Indeed in North American diets there is an abundance of pre-formed vitamin A containing foods including organ meat, beef, eggs, fish oil as well as fortified foods like margarine, non fat milk, breakfast cereal, and some snack foods. In addition, the use of multivitamins/mineral supplements, usually providing 5000 to 10,000 IU (1500-3000 micrograms vitamin A) as retinol or retinyl palmitate (1) is increasingly becoming popular among older population because of their claims being beneficial for many age-related conditions, such as eye health and reduced risk for bladder cancer [10], cataract [11], inflammatory conditions [12, 13], immunity [14] and skin health [15]. In view of the fact that the turnover rate of vitamin $\mathrm{A}$ is slow due to its lipid solubility, detary and supplemental abundance in North American diet and possibly its diminished physiological requirements in old age, may account for its accumulation in the liver over a period of one's life time.

\section{TOXICOLOGICAL IMPLICATIONS OF ELEVATED HEPATIC STORAGE OF VITAMIN A}

The underlying mechanism by which excess vitamin A exerts its toxic effect is not fully understood. It is, however, thought that some of the effects of hypervitaminosis A may be due to labilization of lysosomal membranes [14]. This may occur through a toxic effect on cell membranes, particularly lysosomal membranes. Normally, vitamin A circulates in blood predominantly as free retinol bound to RBP, while in conditions of excessive intake of vitamin $\mathrm{A}$, it is transported in esterified form in association with plasma lipoproteins but not bound to RBP. Vitamin A toxicity appears to occur when the capacity of the protein to transport free retinol is exceeded, so that the vitamin in its esterified form is

Table 2. Age-Associated Changes in Hepatic Total Retinol and Relative Expressions of RBP and cRBP in Male Rats*

\begin{tabular}{|c|c|c|c|}
\hline Age (month) & Retinol (umol/g) & RBP mRNA $^{\mathbf{1}}$ & ${\text { cRBP } \mathbf{m R N A}^{\mathbf{1}}}$ \\
\hline \hline $2-3$ & $362 \pm 25^{\mathrm{a}}$ & $1.0 \pm 0.02^{\mathrm{a}}$ & $1.0 \pm 0.03^{\mathrm{a}}$ \\
\hline $8-10$ & $1037 \pm 54^{\mathrm{b}}$ & $1.0 \pm 0.06^{\mathrm{a}}$ & $0.95 \pm 0.04^{\mathrm{a}}$ \\
\hline $18-20$ & $2134 \pm 37^{\mathrm{c}}$ & $0.69 \pm 0.04^{\mathrm{b}}$ & $1.15 \pm 0.05^{\mathrm{a}}$ \\
\hline
\end{tabular}

*modified from Ref. [9]; each value is the mean of six rats \pm SEM.

${ }^{1}$ for RBPmRNA and cRBPmRNA, the value for 2-3 months old rats was set to 1.0 and other values were expressed relative to this group.

$\mathrm{a}, \mathrm{b}, \mathrm{c}$, letters not commonly shared show significance $(\mathrm{p}<0.05)$. 
carried to the cell membranes by lipoproteins in large quantities. Retinyl esters, because of their amphipathic characteristics, are believed to be more injurious to the membranes via detergent disruption than retinol bound to RBP [15]. It is generally thought that liver vitamin A measure is an indicative of vitamin A toxicity. Since the hepatic store of the vitamin appears to be appreciably high in association with ageing, the possibility of vitamin A toxicity in old age cannot be ignored. It is also noteworthy that in parallel with an age-associated increase in hepatic storage of vitamin A, a progressive increase in the level of this vitamin has been found to occur with ageing of the aortic vascular wall in rats [4]. This age-related changes of vitamin A status is also accompanied by increased productions of a variety of reactive oxygen species in vascular wall [16]. Thus the formations of nitric oxide $\left(\mathrm{NO}^{\circ}\right)$, superoxide $\left(\mathrm{O}_{2}{ }^{-}\right)$and peroxynitrite $\left(\mathrm{ONOO}^{\circ}\right)$ are associated with vascular ageing. It is of further importance that despite an increased NO production, indicated by an increased nitric oxide synthase activity as well as its expression level, free NO release is markedly decreased in aged aorta, when compared with that of the younger rats. This has been suggested to be a consequence of age-associated $\mathrm{O}_{2}{ }^{-}$- production with concomitant quencing of $\mathrm{NO}$ by the formation of $\mathrm{ONOO}^{--}\left(\mathrm{O}_{2}{ }^{--}+\mathrm{NO} \rightarrow \mathrm{ONOO}^{\circ}\right)$. In view of the fact that the ageing process is associated with increased oxidative stress linking to an increased risk for cardiovascular disease, an increasing accumulation of vitamin $\mathrm{A}$ in the liver and the vascular wall with age can be of serious concern.

In recent years, vitamin A toxicity has been suggested to be one of the risk factors for osteoporotic fracture in older population. Thus, there have been numerous animal studies demonstrating that a prolong excessive intake of vitamin A can cause ossification of cartilage, increased bone resorption, extraosseous calcification, hypercalcaemia, and suppressed parathyroid hormone levels [23-25]. More recently, there have been many cross-sectional studies (Table 3), which have compared the dietary intake of retinol and Bone Mineral Density (BMD). In these studies, the retinol intake was estimated from dietary records and a food-frequency questionnaire. Bone Mineral Density was measured with dualenergy x-ray absorptiometry. Hip fracture was identified by using hospital discharge records and was confirmed by record review. According to multivariate analysis of data, retinol intake was negatively associated with BMD. According to a cox regression analysis the risk of fracture was found to be the highest among men with the highest levels of serum retinol in a population-based (2322 men, 49-51 years) longitudinal cohort study [22]. These results are consistent with the results reported from animal and epidemiologic dietary studies.

Crandall [26] re-examined 20 clinical studies; of these 3 were randomized controlled trials, 14 were observational studies, and 3 were case reports. In these studies, retinol intake from diets or supplements has been found to be negatively associated with lumber, femoral neck and trochanter bone mineral density. There is a graded increase in relative risk of hip fracture with increasing intake of retinol, but not ß-carotene intake. Most studies point to a positive relationship between high intake (>1500 ug/d) or high plasma level $(>2.25 \mathrm{umol} / \mathrm{L})$ of vitamin $\mathrm{A}$ and reduced $\mathrm{BMD}$ or an in- creased rate of bone fracture. Indeed, a relationship between hypervitaminosis $\mathrm{A}$ and skeletal malformation and teratogenesis in fetuses have been recognized for many years [27]. On the basis of such recognition, the American College of Obstretricians and Gynaecologists [28] has recommended a maximum dose of $1500 \mathrm{ug} / \mathrm{d}$ prior to and during pregnancy.

Table 3. Studies Reported Fracture Risk $^{1}$ with High Preformed Vitamin A (Retinol) Intake

\begin{tabular}{|c|c|c|}
\hline Reference \# & No. of Subjects & Length of Study \\
\hline \hline$[17]^{*}$ & 1,120 & $64 \mathrm{mo}$ \\
\hline$[18]^{*}$ & 72,337 & $18 \mathrm{yrs}$ \\
\hline$[19]^{*}$ & 958 & $4 \mathrm{yrs}$ \\
\hline$[20]^{* *}$ & 2,332 & $30 \mathrm{yrs}$ \\
\hline$[21]^{*}$ & 2,799 & $22 \mathrm{yrs}$ \\
\hline$[22]^{*}$ & 34,703 & $9.5 \mathrm{yrs}$ \\
\hline
\end{tabular}

${ }^{1}$ bone mineral density (BMD), hip fracture, relative risk.

*postmenopausal women; **men

It is noteworthy that worldwide the highest incidence of osteoporosis occurs in Northern Europe, a population with a high intake of vitamin A. However, a decreased biosynthesis of vitamin D associated with a minimized levels of sun exposure in this population may also contribute to this finding. This has been the basis for some but preliminary studies (to date) to examine the relationship between vitamins A and D in the context of bone metabolism.

\section{DOES VITAMIN A ANTAGONIZES THE ACTION OF VITAMIN D?}

In an experimental study involving weanling male rats, increasing levels of retinyl acetate $(0-8621 \mathrm{ug} / \mathrm{d}$ for $21 \mathrm{~d})$ in a diet eliminated the ability of vitamin $\mathrm{D}$ to elevate the levels of serum calcium while in the absence of the vitamin, these rats maintained a normal serum calcium [29]. These results suggest that vitamin $\mathrm{A}$ in excess antagonized the action of vitamin $\mathrm{D}$ on intestine and bone. A similar effect has been reported in human subjects [30].

Vitamin D is a prohormone. It must be metabolized in order to be activated. The liver and kidney are the main sites for its metabolic activation. Vitamin D is first hydroxylated in the liver at the 25-carbon atom of its molecule to form 25$\mathrm{OH}-\mathrm{D}$; the latter is then further hydroxylated in the kidney at carbon 1-position, forming the active metabolite, 1,25 $(\mathrm{OH})_{2}$-D. The classical actions of this active metabolite are to regulate calcium and phosphate homeostasis and to promote the mineralization of bone [31]. Most of the biological effects occur through the direct transcriptional regulation of specific target genes by binding to the vitamin $\mathrm{D}$ receptor (VDR), which binds to its response element in target genes as a heterodimer with the retinoid receptors, termed retinoid $X$ receptors, PXR [31]. In respect of PAR to influence the activation or expression of appropriate target genes, the preferred receptor partner for heterodimerisation is PXR. Thus, there is potential for interaction between retinoic acid and vitamin D signalling pathways [32, 33]. 


\section{CONCLUSIONS}

Current evidence points to a possible association between an excessive intake of vitamin A, either alone or in combination with low vitamin $\mathrm{D}$, and the risk of osteoporosis. There is, however, little data available on changes of physiological and biochemical properties in animal caused by the interaction between vitamins A and D. Vitamin A could interfere with vitamin $\mathrm{D}$ in terms of its absorption, transport, activation, or vitamin A could stimulate the metabolic degradation of vitamin D. The mechanism of antagonism of vitamin A to vitamin D is far from being clearly understood. Furthermore, it is not yet possible to set a specific level of retinol intake above which bone health is compromised. Based upon the available evidence to date, it is hypothesized that a prolong intake of excess vitamin A affects vitamin D metabolism and that such effect is exacerbated in the presence of a borderline intake of vitamin D.

\section{REFERENCES}

[1] National Academy of Science. Dietary Reference Intake: Recommended Intakes for Individuals. Food and Nutrition Board, Institute of Medicine, 2004

[2] Ross AC. Overview of retinoid metabolism. J Nutr 1993; 123: 34650.

[3] Groff JL, Gropper SS. Fat soluble vitamins. In: Advanced Nutrition and Human Metabolism, Wadsworth, CA, USA. 1999, pp. 316-370.

[4] van der Loo B, Labugger R, Aebischer CP, et al. Age-related changes of vitamin A status. J Cardiovasc Pharmacol 2004; 43: 2630 .

[5] Raica NJr, Scott J, Lowry L, Sauberlich HE. Vitamin A concentration in human tissues collected from five areas in the United States. Am J Clin Nutr 1972; 25: 291-6.

[6] Ross AC, Zofaghari R. Regulation of hepatic retinol metabolism: Perspectives of studies on vitamin A status. J Nutr 2004; 134(suppl): 269-275.

[7] Tyler H, Dickerson JWT. Determination of serum retinol in cancer studies. Eur J Cancer Clin Oncol 1984; 20: 1205-6.

[8] Smith FR, Goodman DS. Retinol-binding protein and regulation of vitamin A transport. Fed Proc 1979; 38(11): 2504-9.

[9] Dawson HD, Yamamoto Y, Zolfaghari R, et al. Regulation of hepatic vitamin A storage in a rat model of controlled vitamin A status during aging. J Nutr 2000; 130: 1280-6.

[10] Steinmaus CM, Nunez S, Smith AH. Diet and bladder cancer - a meta analysis of six dietary vegetables. Am J Epidemiol 2000; 157: 693-702.

[11] Owsley C, McGwin G, Jackson GR, et al. Effect of short-term, high-dose retinol on dark adaptation in aging and early age-related maculopathy. Investig Ophthal Visual Sci 47: 2006; 1310-8.

[12] Rahman MM, Mahalanabis D, Hossain S, et al. Simultaneous vitamin A administration at routine immunization contact enhances antibody response to diptheria vaccine in infants younger than six months. J Nutr 1999; 129: 2192-5.

[13] Bahl R, Bhandari N, Kant S, malbak K, Ostegaard E, Bhan MK. Effect of vitamin A administration at expanded program on immunization contacts on antibody response to oral polio vaccine. Eur J Clin Nutr 2002; 56: 321-5.

[14] Zile MH, Callun ME. The function of vitamin A: Current concepts. Proc Soc Exp Biol Med 1983; 172: 139-52.

[15] Howard WB, Willhite CC. Toxicity of retinoids in humans and animals. J Toxicol Toxin Rev 1986; 5: 55-94.

[16] van der Loo B, Labugger R, Skepper JN, et al. Enhanced peroxynitrite formation is associated with vascular aging. J Exp Med 2000; 192: 1731-43.

[17] Melhus H, Michaelsson K, Kindmark A, et al. Excessive dietary intake of vitamin $\mathrm{A}$ is associated with reduced bone mineral density and increased risk of hip fracture. Ann Internal Med 1998; 129: 770-8.

[18] Feskanich D, Singh V, Willet WC, Colditz GA. Vitamin A intake and hip fractures among postmenopausal women. J Am Med Assoc 2002; 287: 47-54.

[19] Promislow JHE, Goodman-Gruen D, Slymen DJ, Barrett-Connor E. Retinol intake and bone mineral density in the elderly: the Rancho Bernardo Study. J Bone Miner Res 2002; 17: 1349-58.

[20] EreMichaelsson MD, Lithell H, Vessby B, Melhus H. Serum retinol levels and risk of fracture. N Engl J Med 2003; 348: 287-94.

[21] Opotowsky AR, Bilezikian JP, NHANES I follow-up study. Serum vitamin A concentration and the risk of hip fracture among women 50 to 74 years old in the United States: A prospective analysis of the NHANES 1 follow-up study. Am J Med 2004; 117: 169-74.

[22] Lim LS, Harnack LJ, Lazovich D, Folsom AR. Vitamin A intake and the risk of hip fracture in postmenopausal women: The Iowa Women's Health Study. Osteoporos Int 2004; 15: 552-9.

[23] Moor T, Sharma IM. Hypervitaminosis A combined with calcium deficiency in rats. Int J Vitam Nutr Res 1979; 49: 14-20.

[24] Frankel TL, Seshadri MS, McDowall DB, Cornish CJ. Hypervitaminosis A and calcium-regulating hormones in the rat. J Nutr 1986; 116: 578-87.

[25] Hough S, Avioli LV, Muir H, et al. Effects of hypervitaminosis A on the bone and mineral metabolism of the rat. Endocrinology 1988; 122: 2933-66.

[26] Crandall C. Vitamin A intake and osteoporosis: A clinical review. J Women's Health 2004; 13: 939-53.

[27] Public Affairs Committee of Teratology Soc. Position paper: Recommendations for vitamin A use during pregnancy. Teratology 1987; 35: 269-75.

[28] American College of Obstetricians and Gynecologists (ACOG) Committee: Maternal and Fetal Medicine. Vitamin A supplementation during pregnancy. Int J Gynecol Obstet 1993; 40: 175-8.

[29] Rohde CM, Manatt M, Clagett-Dame M, DeLuca HF. Vitamin A antagonizes the action of vitamin D in rats. J Nutr 1999; 129: 2246 50 .

[30] Johansson SMH. Vitamin A antagonizes calcium response to vitamin D in man. J Bone Miner Res 2001; 16: 1899-905.

[31] Feldman DP, Malloy J, Gross C. Vitamin D metabolism and action. In: R. Marcus, D Feldman and J Kelsey, eds. Osteoporosis, San Diego, Academic Press 1996; pp. 205-235.

[32] Glass CK. Differential recognition of target genes by nuclear receptor monomers, dimers, and heterodimers. Endocr Rev 1994; 15: 391-407.

[33] Carlberg C. Mechanisms of nuclear signalling by vitamin D3: Interplay with retinoid and thyroid hormone signalling. Eur $\mathrm{J}$ Biochem 1995; 231: 517-27. 